\title{
STUDIES ON THE ANTIOXIDANT ACTIVITY OF THE COMPOUND ENOXIL AND ITS RELATED FORMS
}

\author{
Tudor Lupaşcu*, Alexandru Gonța \\ Institute of Chemistry, Academy of Sciences of Moldova, \\ 3 Academiei str., MD 2028, Chisinau, Republic of Moldova \\ E-mail: lupascut@gmail.com
}

\begin{abstract}
Studies of the antioxidant activity of polyphenolic compounds extracted from grape seeds are of considerable interest currently. The compound Enoxil is a mixture of polyphenols, esthers, and carboxylic acids, which could be involved in reducing the risk of diseases associated with oxidative stress. The results of the investigation of antioxidant activity for the compound Enoxil and its forms are presented in this paper. Two distinct methods were used to assess the antioxidant properties of the tested compounds: the radical monocation of 2,2'-azinobis-(3-ethylbenzothiazoline6-sulfonic acid) (ABTS ${ }^{++}$test) and the DPPH method (2,2-diphenyl-1-picrylhydrazyl radical). It can be concluded that acetone-Enoxil has a higher antioxidant activity that other tested compounds.
\end{abstract}

Keywords: polyphenols, antioxidants, DPPH', ABTS+.

\section{Introduction}

A current global problem is the negative influence on the human body of various pathogenic diseases, mutagenic and psychological factors. Among the psychological states that lead to human health disorder is stress, which is a major factor in the emergence of various diseases and abnormalities [1,2].

Scientist study the causes and factors responsible for these effects in the body, to interfere and prevent their harmful action.

One of the causes of disturbances in the body at biological, chemical, genetic and psychological level is the formation of free radicals and oxidative stress [3]. Air pollution, excessive radiation and toxic waste are sources of "primary" radicals derived from the environment. For example, large quantities of free radicals are generated in photolysis processes and water radiolysis [4].

Thus, an antioxidant is a substance that at extremely low concentrations, as compared to the substrate, prevents or significantly delays its oxidation. Antioxidants work properly in close correlation with the structure of free radicals, the harmful agent concentration, with the properties and mode of action in target tissues [5].

Flavonoids are a large class of compounds present in plants, which possess antioxidant properties. These compounds contain phenolic hydroxyl groups in the ring structure, which offer them reducing properties.

The antioxidant activity of polyphenols is determined by the presence of hydroxyl groups in the $3^{\prime}$ and 4 ' positions of the B ring and to a lesser extent of the $4^{\prime}$ hydroxyl group in ring B [6]. Flavonols, especially catechins, quercitin, kaemferol and glucosides, are constituents of green and black teas [7], red wine and grape seeds.

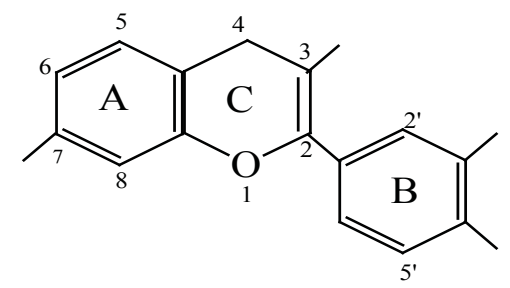

Fig. 1. General chemical structure of flavones [6]

In vitro studies defined the antioxidant potential of polyphenols as a parameter that determines the ability to capture free radicals such as superoxide radicals, singlet oxygen, hydroxyl radical, peroxyl radical (which induce various diseases). Chemical structures that contribute to the antioxidant activity of polyphenols, including a neighboring dihydroxy- or trihydroxy- structure, may chelate metal ions by forming complexes and prevent the generation of free radicals. This structure also allows electron delocalization, conferring high reactivity in free radicals quenching [8].

There is an increasing interest in using natural compounds, such as polyphenols extracted from grape seeds. Various extraction systems were tried to obtain new products such as tannins. Antioxidant, antibacterial and antifungal action exhibited by these compounds is widely studied in the literature. Also due to low solubility in water or alcohol, instability such as oxidation or reduction, these compounds cause problems in the formulation of new drugs [9].

Grape seeds contain a wide range of polyphenolic compounds such as enotannins. In order to improve the 
physicochemical properties of these compounds, their structure and physico-chemical properties were modified by oxidation reactions, which resulted in obtaining a new product, Enoxil [10-12].

The obtained preparation presents scientific and practical interest, since manufacturing this product is performed from natural raw materials, sustainable and safe and, unlike other drugs, it does not generate side effects.

Use of antioxidants in cosmetics and pharmaceuticals is widespread. The study of antioxidant properties of the compound Enoxil, used to formulate new cosmetics, has a great importance in stabilizing lipid oxidative processes and other active forms.

For the in vitro evaluation of antioxidant capacity of the isolated compounds, or mixture of compounds, different methods are used, involving the use of various mechanisms for determining the antioxidant activity. The most popular are: the $\mathrm{ABTS}^{\cdot+}$ test (2,2'-azinobis-(3-ethyl-6-sulphonate benzothiazoline) radical cation) [13], the test DPPH' (2,2-diphenyl1-picrylhydrazyl) [14] and the FRAP test, based on the reduction of the ferric tripyridyltriazine complex to the ferrous form to test the total reduction capacity of the antioxidant [15].

\section{Experimental}

In our research, we used two methods to determine the antioxidant activity, namely the $\mathrm{DPPH}^{\bullet}$ test and the $\mathrm{ABTS}^{\bullet+}$ test.

\section{Reagents for the DPPH method}

\begin{tabular}{|l|l|}
\hline Reagent & Chemical formula \\
\hline DPPH radical, D9132, Aldrich & 2,2-Diphenyl-1-picrylhydrazyl \\
\hline Ethanol solution & $\mathrm{C}_{2} \mathrm{H}_{5} \mathrm{OH}$ \\
\hline
\end{tabular}

\section{Reagents for the ABTS method}

\begin{tabular}{|l|l|}
\hline Reagent & Chemical formula \\
\hline ABTS radical & $\mathbf{2 , 2}$-azino-bis(3-ethylbenzthiazoline-6-sulphonic acid \\
\hline $\begin{array}{l}\text { Na persulfate } \\
\text { S6172, Sigma, }>98 \% \text { BioXtra }\end{array}$ & $\mathrm{Na}_{2} \mathrm{~S}_{2} \mathrm{O}_{8}$ \\
\hline Ethanol solution & $\mathrm{C}_{2} \mathrm{H}_{5} \mathrm{OH}$ \\
\hline
\end{tabular}

Table 1

\section{Enoxil fractions}

\begin{tabular}{|l|l|}
\hline Fraction & Color \\
\hline Enoxil standard & Yellow \\
\hline Enoxil - ethanol fraction & Yellowish brown \\
\hline Enoxil - acetone fraction & Dark yellows \\
\hline Enoxil - ethylacetate fraction & Pale yellow \\
\hline
\end{tabular}

Various concentrations were prepared of Enoxil and its forms, in the range $3000-15000 \mu \mathrm{g} / \mathrm{mL}$ and in the final solution to be analyzed (using the ABTS method), the concentrations ranged between 30 and $150 \mu \mathrm{g} / \mathrm{mL}$.

The $\mathrm{ABTS}^{\cdot+}$ radical cation is formed by the reaction of $\mathrm{ABTS}^{\cdot+}(7 \mathrm{mM}$ solution) and potassium persulfate $(2.45 \mathrm{mM}$, final concentration) for 12-16 hours in the dark at room temperature. The obtained ABTS ${ }^{+}$solution $\left(2,2^{\prime}\right.$-azino-bis (3ethylbenzthiazoline-6-sulfonic acid)) is diluted with 70\% ethanol to give an absorbance of $0.700 \pm 0.020$ at $734 \mathrm{~nm}$. The

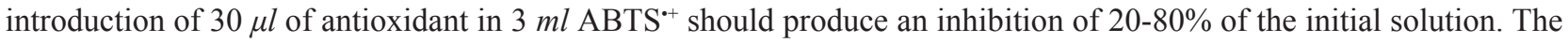
calibration curve is graphed using Trolox standard (0-15 mM range of concentrations) absorbance readings at $734 \mathrm{~nm}$, at 1 and 6 min after mixing [13].

The analysis of antioxidant activity by DPPH method was performed using $3.5 \mathrm{ml}$ of ethanol (70\%) solution of $\mathrm{DPPH}^{\cdot}$ with the initial concentration of $60 \mu \mathrm{M}$, to which was added $0.5 \mathrm{ml}$ antioxidant solution with different concentrations (final concentrations were between 30-150 $\mu \mathrm{g} / \mathrm{mL}$ ) and the optical density at $517 \mathrm{~nm}$ was measured at 1 , $5,10,20,30,60$ and 120 minutes with T80 spectrophotometer in $1 \mathrm{~cm}$ quartz cuvettes [14].

\section{Results and discussions}

Antioxidant properties of the preparation Enoxil were studied using the DPPH test analysis and the interaction with the $\mathrm{ABTS}^{++}$radical cation, the results are presented in tables and figures below. We studied the antioxidant properties of different Enoxil fractions obtained in the laboratory. According to the method 
for determining the antioxidant activity using $\mathrm{ABTS}^{\bullet+}$, the consumption of $\mathrm{ABTS}^{\cdot+}$ was recorded using the absorbance values after $1 \mathrm{~min}$ and $6 \mathrm{~min}$. Using the obtained data, were potted kinetic graphs of ABTS ${ }^{\cdot+}$ reduction at its interaction with different antioxidant fractions of Enoxil used for analysis (fig.1-4).

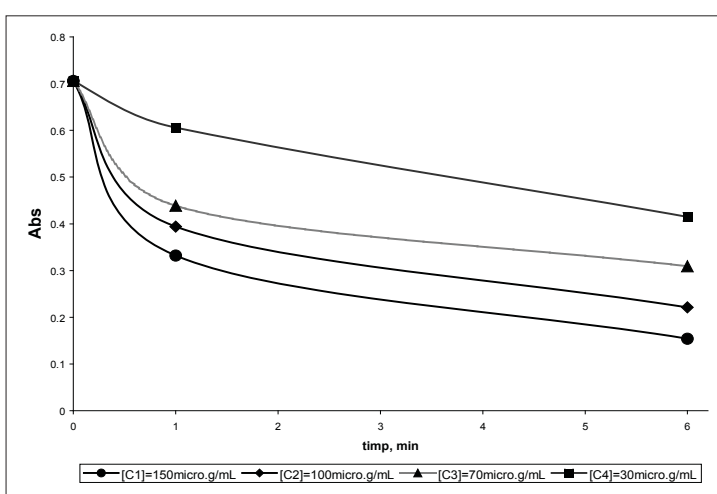

Fig. 1. Reduction of $\mathrm{ABTS}^{++}$with Enoxil-ethylacetate fraction

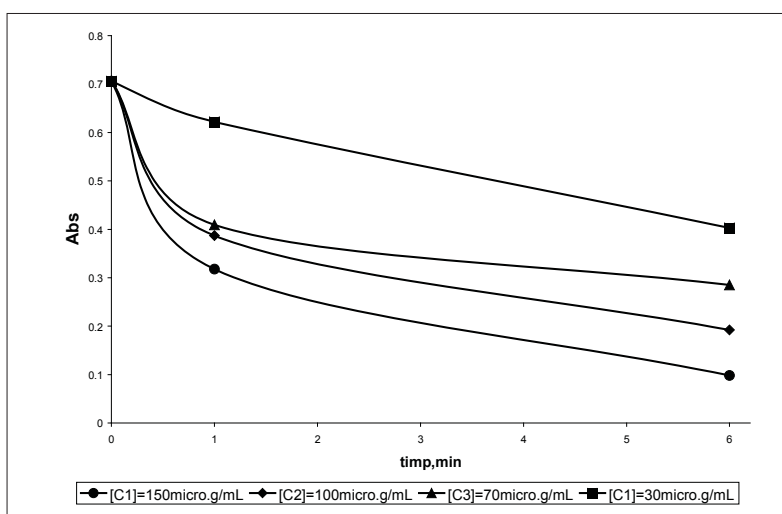

Fig. 3. Reduction of $\mathrm{ABTS}^{\cdot+}$ with Enoxil-acetone fraction

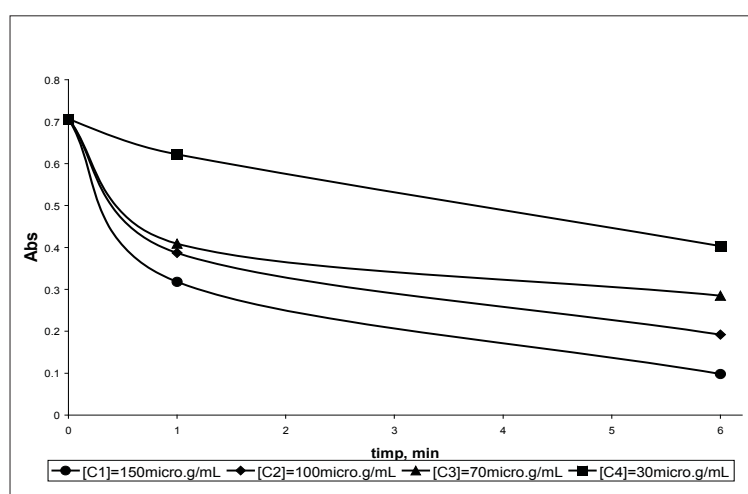

Fig. 2. Reduction of $\mathrm{ABTS}^{++}$with Enoxil-ethanol fraction

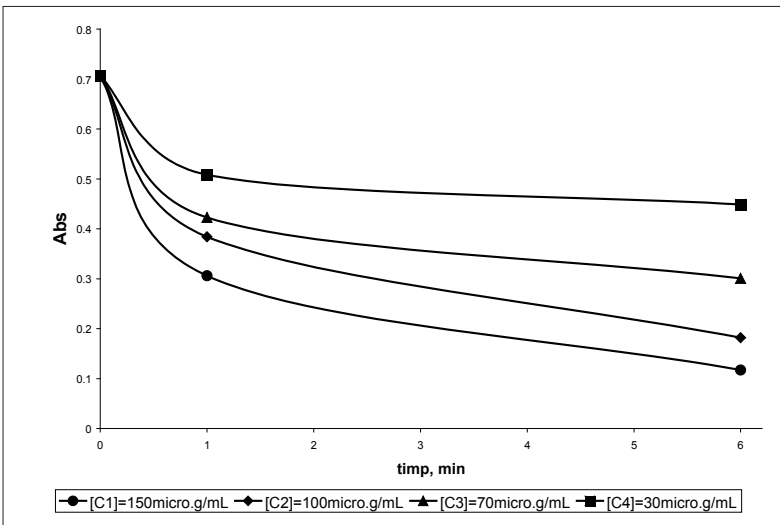

Fig. 4. Reduction of $\mathrm{ABTS}^{\circ+}$ with standard Enoxil

According to the protocol, the antioxidant activity in Trolox equivalents was calculated. For this purpose, Trolox solutions were prepared of known concentrations, which were subjected to oxidation with ABTS, as specified above antioxidant samples. In the next stage, the obtained kinetic experimental data were used to plot graphs (fig. 5) and antioxidant activities were calculated in Trolox equivalents for the studied compounds solutions.

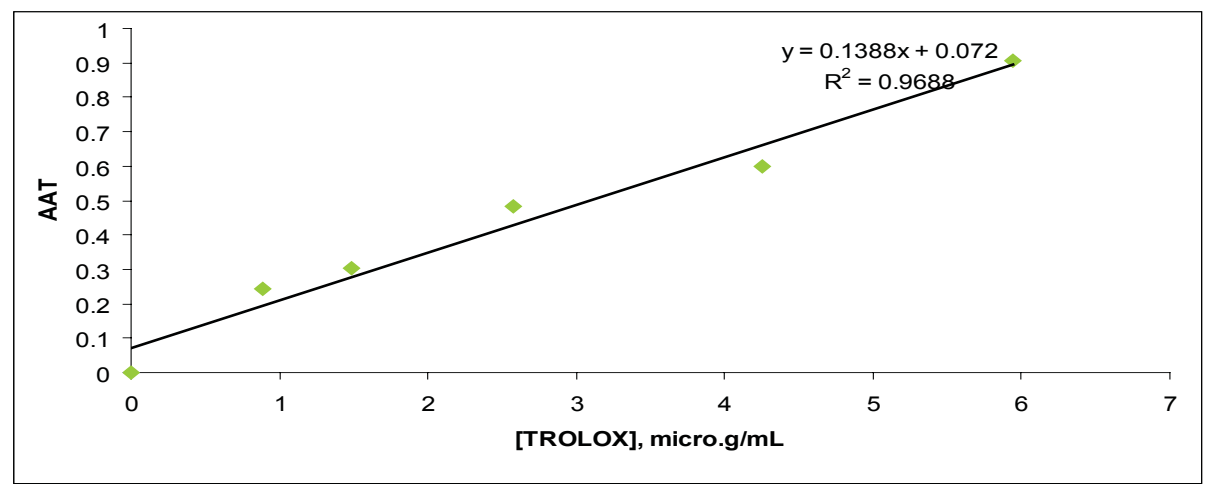

Fig. 5. Total antioxidant activity as function of $[\mathrm{Tx}]_{0}$, determined by the $\mathrm{ABTS}^{++}$test 
Dependence of the antioxidant activity, in Trolox equivalents, on the Enoxil-ethyl acetate concentration

\begin{tabular}{|c|c|c|}
\hline Concentration, $\boldsymbol{\mu g} / \mathbf{m L}$ & AAT & Equiv-TROLOX \\
\hline 150 & 0.521246 & 3.168 \\
\hline 100 & 0.433428 & 2.554 \\
\hline 70 & 0.369688 & 2.090 \\
\hline 30 & 0.260623 & 1.326 \\
\hline
\end{tabular}

Table 3

Dependence of the antioxidant activity, in Trolox equivalents, on the Enoxil-ethanolic concentration

\begin{tabular}{|c|c|c|}
\hline Concentration, $\boldsymbol{\mu g} / \mathbf{m L}$ & AAT & Equiv-TROLOX \\
\hline 150 & 0.538244 & 3.297 \\
\hline 100 & 0.443343 & 2.623 \\
\hline 70 & 0.398017 & 2.307 \\
\hline 30 & 0.264873 & 1.356 \\
\hline
\end{tabular}

Dependence of the antioxidant activity, in Trolox equivalents, on the Enoxil-acetone concentration

\begin{tabular}{|c|c|c|}
\hline Concentration, $\boldsymbol{\mu g} / \mathbf{m L}$ & AAT & Equiv -TROLOX \\
\hline 150 & 0.541076 & 3.316 \\
\hline 100 & 0.443343 & 2.623 \\
\hline 70 & 0.412181 & 2.406 \\
\hline 30 & 0.252125 & 1.257 \\
\hline
\end{tabular}

Dependence of the antioxidant activity, in Trolox equivalents, on the standard Enoxil concentration

\begin{tabular}{|c|c|c|}
\hline Concentration, $\boldsymbol{\mu g} / \mathbf{m L}$ & AAT & Equiv -TROLOX \\
\hline 150 & 0.531074 & 3.271 \\
\hline 100 & 0.447592 & 2.582 \\
\hline 70 & 0.392351 & 2.282 \\
\hline 30 & 0.271955 & 1.428 \\
\hline
\end{tabular}

The obtained experimental results show that AAT depends on the concentration of the analyzed Enoxil forms and for the studied concentration range, AAT varies within the range $0.55-0.26$. The lowest antioxidant activity was observed for the form obtained by ethyl acetate extraction (concentration $150 \mu \mathrm{g} / \mathrm{mL}$ ).

From the obtained data we can conclude that for the maximal tested concentration, i.e. $150 \mu \mathrm{g} / \mathrm{mL}$ of antioxidant, the effectiveness of antioxidant activity in the studied fractions decreases in the order: ethyl acetate $<$ standard Enoxil $<$ Enoxil-ethanol fraction $<$ Enoxil-acetone fraction.

Enoxil antioxidant properties are comparable with the antioxidant capacities of catechin, epicatechin, epigalocatechin and others [16].

It is known that mentioned above antioxidants are widely used in experimental research, such as inhibition of cancer development, stopping the cells aging process, protection and regeneration of skin [17].

In general, it is known that the form of obtained kinetic graphs is determined by the molecular structure of the compound under study, involving the stability of the antioxidant radical. Since the molecular structures of Enoxil forms are similar, one would expect similar kinetic graphs.

In polyphenols, the presence of the dihydroxo- group in positions $3^{\prime}$ and $4^{\prime}$ of $\mathrm{B}$ ring favors reactions with slow kinetic behavior. However, the presence of compounds with other structures involves a behavior characterized by expression of two stages, the first being fast and the other slow (fig.6), depending on the extraction method. 


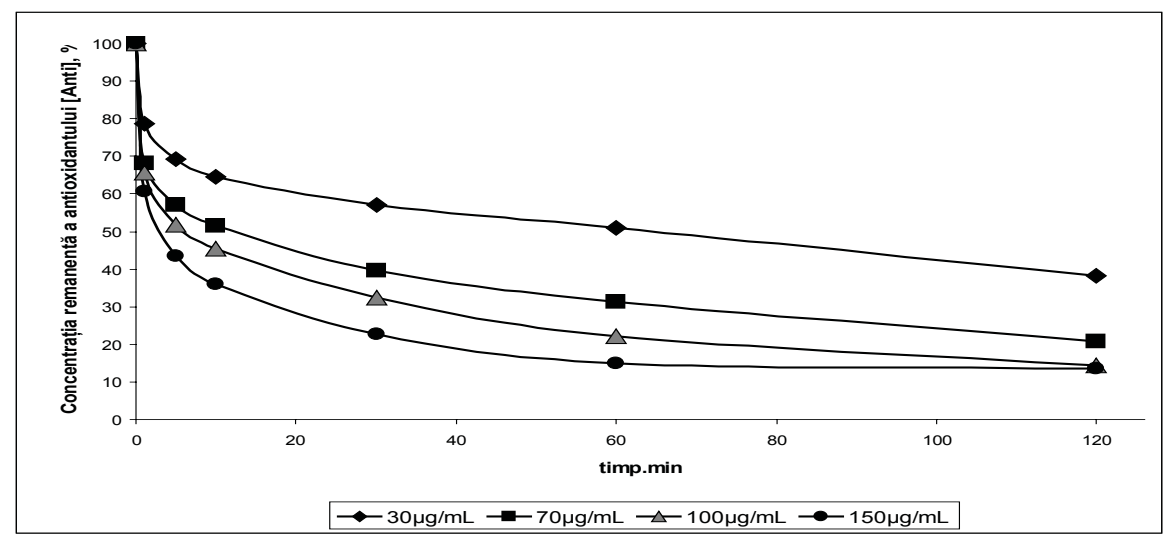

Fig. 6. Kinetic graphs of reduction of Enoxil-ethanol (various concentrations) with DPPH*

The antioxidant/antiradical activity is defined as the antioxidant amount necessary to decrease the initial DPPH concentration by $50 \%$. It is called the $50 \%$ effective concentration $\left(\mathrm{EC}_{50}\right)$. For simplicity we used the value $1 / \mathrm{EC}_{50}$ called the antiradical power (PAR) or antiradical activity. Thus, the higher is the PAR value, the more effective is the antioxidant. The antiradical activity is determined from the dependence graph of remaining [DPPH'] (in percent) function of the molar ratio (MR) or mass ratio of the antioxidant and DPPH' $\left(\mathrm{RM}=[\mathrm{ANTI}]_{0} /[\mathrm{DPPH}]_{0}\right)$. Since the antioxidant activity of test compounds is characterized by two-stage kinetics, the antioxidant activity was determined at the equilibrium. Fig.7 and 8 show the results of experimental determination of $\mathrm{EC}_{50}$ for various fractions.

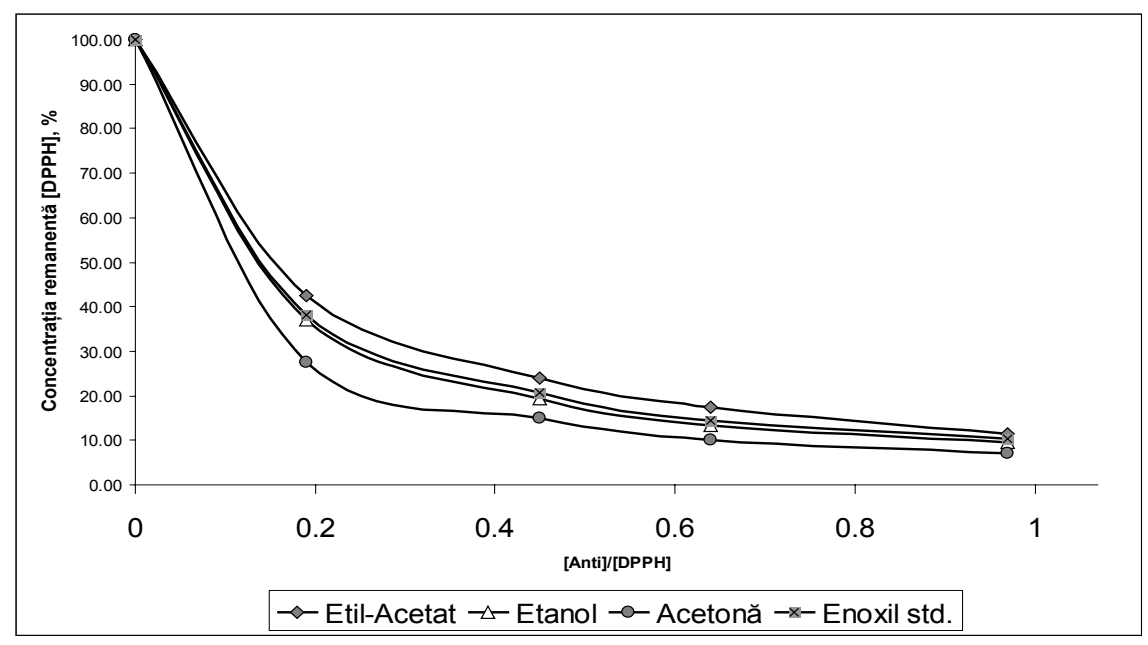

Fig. 7. Kinetic graphs of [DPPH] reduction, \% function of the ratio [Anti]/[DPPH]

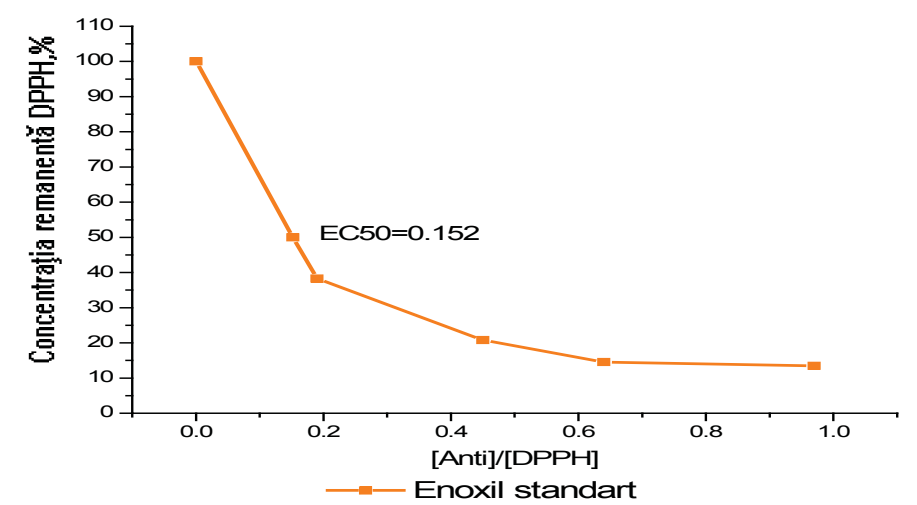

Fig. 8. Kinetic graph of [DPPH'] reduction, \% function of the ratio [Anti]/[DPPH]

Thus Enoxil-acetone fraction has the highest antioxidant activity in the reaction with DPPH* $(\mathrm{PAR}=7.7)$, giving a molar ratio $\mathrm{EC}_{50}$ of 0.13 . 


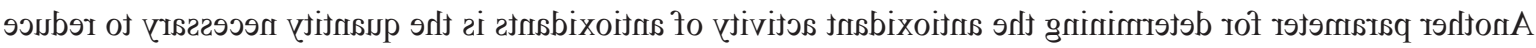

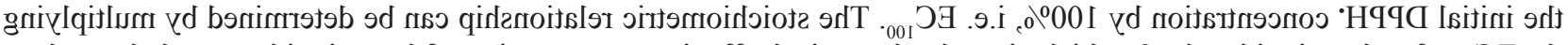

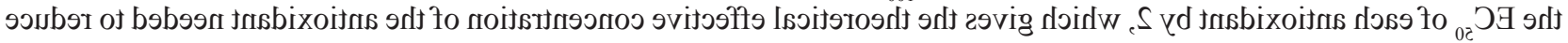

:Н

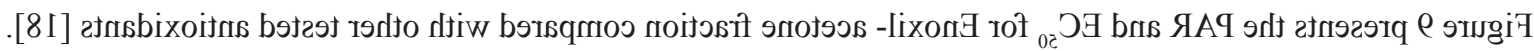

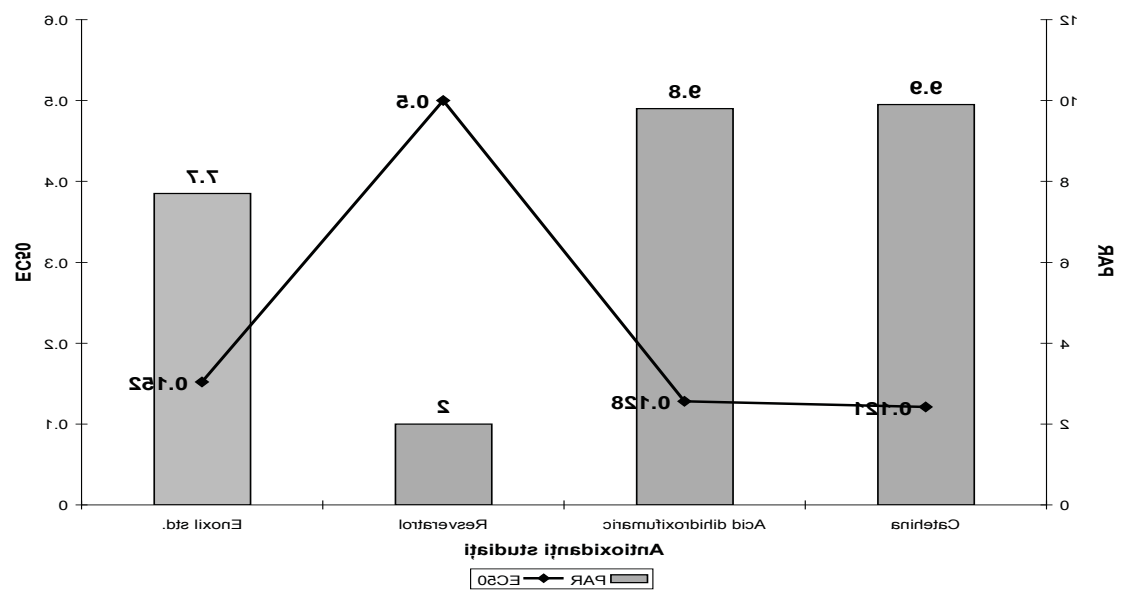

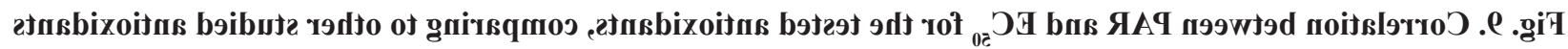

anoizalonoכ

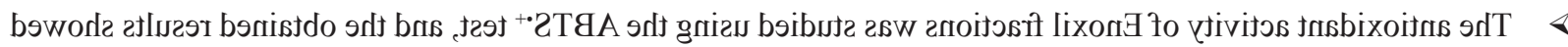

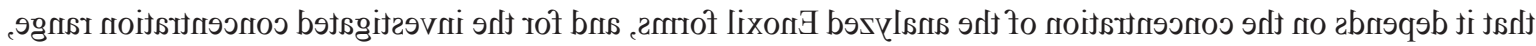

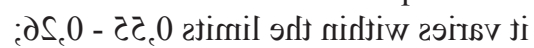

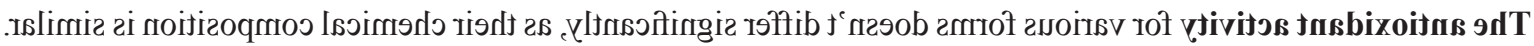

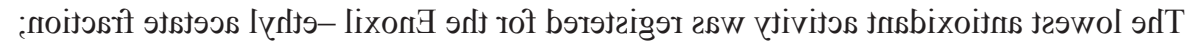
эnt enrsbixoitns to JmI

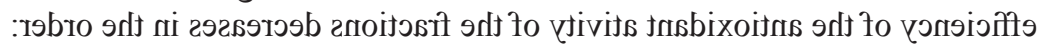

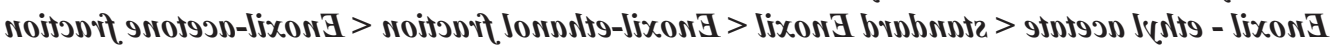

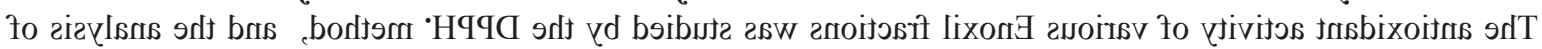

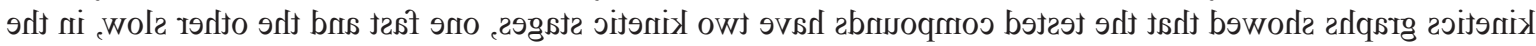

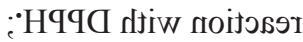

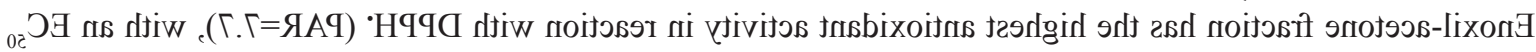
.esi,0 to эulsv

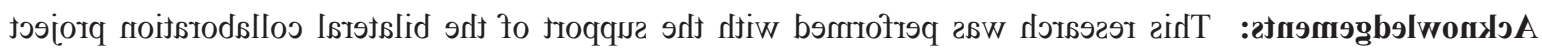

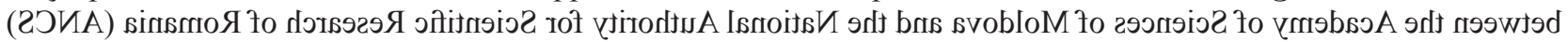

.А०Я 181. ¿0.0S8.0I .Oก

\section{ә9эกรังตรด}

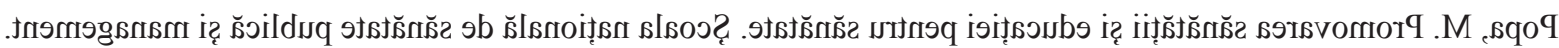

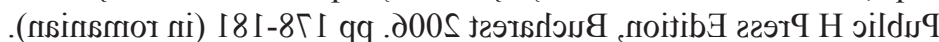

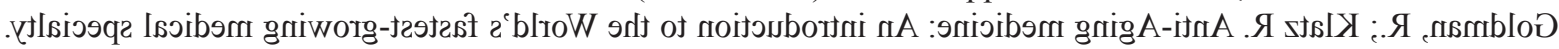

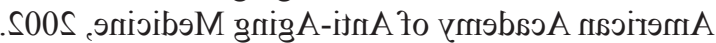

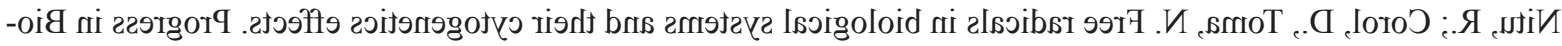

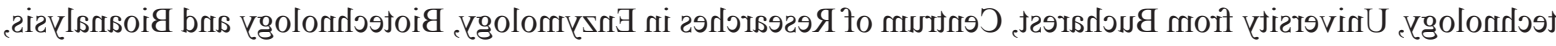

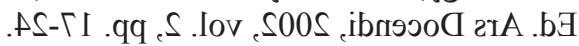

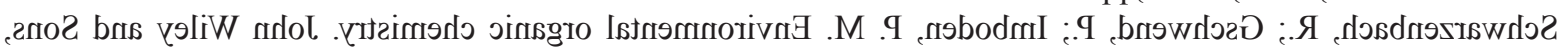

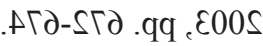

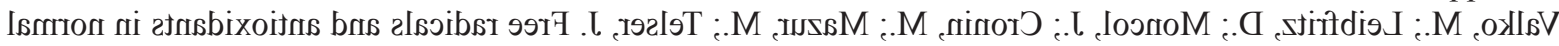

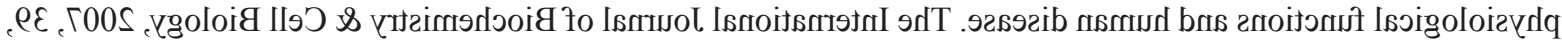

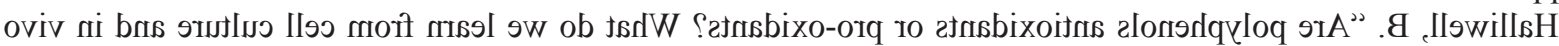

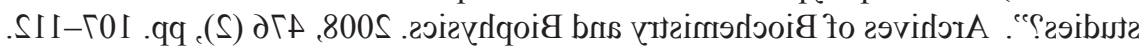

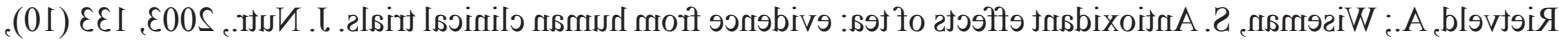


[8]. Stohs, S.; Bagchi, D. Oxidative mechanisms in the toxicity of metal ions. Free Radical Biology and Medicine, 2005, 18 (2), pp. 321-36.

[9]. Gonta, A.; Bobeico, V. Activitatea antioxidanta a polifenolilor. Abstract of communication. National scientific conference for young researchers, 10 Edition, Chisinau, 2006, pp. 93-94 (in romanian).

[10]. Chung, K.; Lu, Z.; Chou, M. Mechanism of inhibition of tannic acid and related compounds on the growth of intestinal bacteria. Food and Chemical Toxicology, 1998, 36(12), pp. 1053-1060.

[11]. Kulciţki, V.; Vlad, P.; Duca, Gh. Investigation of grape seed proantocyanidins. Chemistry Journal of Moldova, 2007, 2(1), pp. 36-50.

[12]. Lupascu, T.; Lupascu, L. The obtaining procedure of the water soluble enotannins. MD Patent Nr. 3125. BOPI: 8, 2006.

[13]. Van den Berg, R.; Haenen, G. R.; Van den Berg, H.; Van der Vijgh, W.; Bast, A. The predictive value of the antioxidant capacity of structurally related flavonoids using the Trolox equivalent antioxidant capacity (TEAC) assay. Food Chem., 2000, 70, pp. 391-395.

[14]. Sroka, Z.; Cisowski, W. Hydrogen peroxide scavenging, antioxidant and anti-radical activity of some phenolic acids. Food Chem. Toxicol. 2003, 41, pp. 753-758.

[15]. Benzie, I. F.; Strain, J. J. The ferric reducing ability of plasma (FRAP) as a measure of "antioxidant power", the FRAP assay. Anal. Biochem., 1996, 239, pp. 70-76.

[16]. Muselík, J.; García-Alonso, M.; Martín-López, M.; Žemlička, M.; Rivas-Gonzalo, J. C. Measurement of antioxidant activity of wine catechins, procyanidins, anthocyanins and pyranoanthocyanins. Int. J. Mol. Sci., 2007, 8, pp. 797-809.

[17]. Diana-Simona, A.; Gabriela, G.; Zeno, G. The anthocyans: Biologically active substances of food and pharmaceutical interest. The Annals of University Dunarea de Jos, Romania, Fascicle IV-Food Technology, 2003, pp. 106-115.

[18]. Porubin, D. Utilizarea compusilor naturali extrasi din produse secundare vinicole in inhibitia N-Nitrozoaminelor. $\mathrm{PhD}$ thesis abstract, Chisinau, 2009 (in romanian). 